\title{
A self-adaptive distance regularized level set evolution method for optical disk segmentation $^{1}$
}

\author{
Huiqun $\mathrm{Wu}^{\mathrm{a}}$, , Xingyun Geng ${ }^{\mathrm{a}}$, Xiaofeng Zhang ${ }^{\mathrm{a}}$, Mingyan Qiu ${ }^{\mathrm{a}}$, Kui Jiang ${ }^{\mathrm{a}}$, Lemin Tang ${ }^{\mathrm{c}}$, \\ Jiancheng Dong, ${ }^{\mathrm{a}, *}$ \\ ${ }^{a}$ Department of Medical Informatics, Medical School of Nantong University, Jiangsu 226001, China \\ ${ }^{b}$ Key Laboratory of Medical Imaging Computing and Computer Assisted Intervention (MICCAI) \\ of Shanghai, Fudan University, Shanghai 200032, China \\ ${ }^{c}$ Department of Medical Image Engineering, Medical School of Nantong University, Jiangsu \\ 226001, China
}

\begin{abstract}
The optic disc (OD) is one of the important anatomic structures on the retina, the changes of which shape and area may indicate disease processes, thus needs computerized quantification assistance. In this study, we proposed a self-adaptive distance regularized level set evolution method for OD segmentation without the periodically re-initializing steps in the level set function execution to a signed distance function during the evolution. In that framework, preprocessing of an image was performed using Fourier correlation coefficient filtering to obtain initial boundary as the beginning contour, then, an accurate boundary of the optic disc was obtained using the self-adaptive distance regularized level set evolution method. One hundred eye fundus color numerical images from public database were selected to validate our algorithm. Therefore, we believe that such automatic OD segmentation method could assist the ophthalmologist to segment OD more efficiently, which is of significance for future computer-aided early detection of glaucoma and retinopathy diseases.
\end{abstract}

Keywords: Optic disk, retinal imaging, level set evolution, imaging informatics

\section{Introduction}

A retinal image is an important interface for various eye diseases' diagnosis, and its analysis and processing has great significance in the field of clinical medicine, especially in non-invasive chronic disease detecting and monitoring. The optic disc (OD) is one of the important anatomic structures on the retina at which optic nerve axons enter and leave the eye. Changes of OD shape and area may indicate many disease processes, particularly in glaucoma, diabetic retinopathy (DR), age-related

\footnotetext{
${ }^{1} \mathrm{HW}$ and $\mathrm{XG}, \mathrm{XZ}$ conceived the study, evaluated the algorithm and wrote the manuscript. HW, MQ implemented the algorithm. KJ, MQ performed the experiments. LT and JD conceived the experiments. All authors read and approved the final manuscript.

*Corresponding author: Jiancheng Dong, Department of Medical Informatics, Medical School of Nantong University, Jiangsu 226001, China. Tel.: 86-513-85051891; Fax: 86-513-85051820; E-mail: dongjc@ntu.edu.cn.
}

0959-2989/14/\$27.50 @ 2014 - IOS Press and the authors. 
macular degeneration (ARMD), parapapillary atrophy and glaucoma and accurate identification of the OD boundary may be used to quantify such changes [1-5].

Previous studies have approached different segmented and quantification methods for OD on retinal fundus images [6-9]. Properties such as a circular structure and high intensity of the optic disc are widely considered as important features for further OD classification. Zheng et al proposed a fairly general energy function that can naturally fit into a global optimization framework with graph cut to automatically segmentation of OD and cup from color fundus photograph. In their study, an energy function includes priors on the shape, disc location, geometric features together with the effective optimization of graph cut were performed [10]. Cemal et al extracted intensity and textural features from a retinal image, and proposed simple and efficient statistical methods for the detection, segmentation, and measurement of the OD [11].

Given that the mixture of retinal vessel and OD pixels on the OD region, the effects of clustering methods and histogram-based methods are limited. However, the contour-based method has its special advantage in avoiding above-mentioned issues. Early geometric active contour-based models were typically based on partial differential equation (PDE) driven level set methods, representing contours as the zero level set of an implicit function defined in a higher dimension. In implementing such methods, it is numerically in need of re-initialization steps to overcome irregularities during the level set evolution process [12].

In this study, we proposed a self-adaptive distance regularized level set evolution method for OD segmentation without the periodically re-initializing steps in the level set function execution to a signed distance function during the evolution. The proposed algorithm consists of two steps-in the first step, preprocessing of an image was performed using Fourier correlation coefficient filtering to obtain initial boundary as the beginning contour; while in the second step, accurate boundary of optic disc was formed using level set method. In the following sections, we described our approach and validated its efficiency in public dataset in comparison of other reported approaches.

\section{Methods}

\subsection{Dataset}

In this study, 100 eye fundus color numerical images (Subset Base31) were obtained from the publicly available Messidor database (http://messidor.crihan.fr) which was acquired by three ophthalmologic departments using a color video 3CCD camera on a Topcon TRC NW6 non-mydriatic retinograph with a 45 degree field of view. The images were obtained using eight bits per color plane at $1440 * 960,2240 * 1488$ or $2304 * 1536$ pixels, with pupil dilation (one drop of Tropicamide at $0.5 \%$ ). All images were stored in TIFF format and an Excel file with medical diagnoses' annotation for each image. Besides, another 100 manually segmented OD binary images were prepared by a clinician and were treated as ground truth (Figure 1).

In this study, we tested the system on Intel Pentium duo core CPU P6000, Windows 7 Home Basic operating system and MATLAB (R2012a).

\subsection{Workflow}

In this study, a retinal image was firstly transformed into frequency field, in which the OD region was roughly located using Fourier correlation coefficient filtering. Then, an initial OD boundary was 

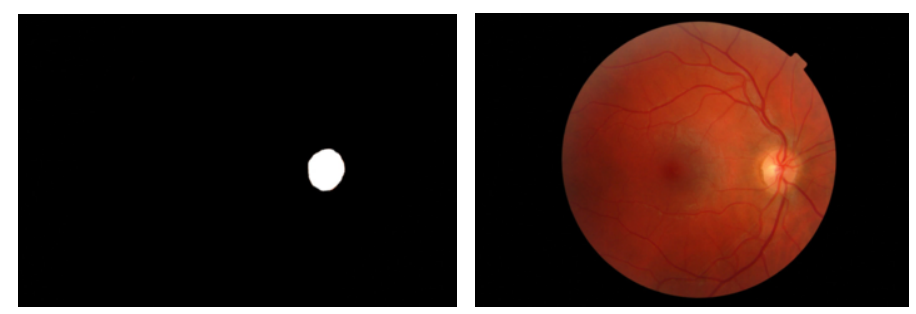

Fig. 1. The manually OD segmentation results (left) and correspondent tested image (right).

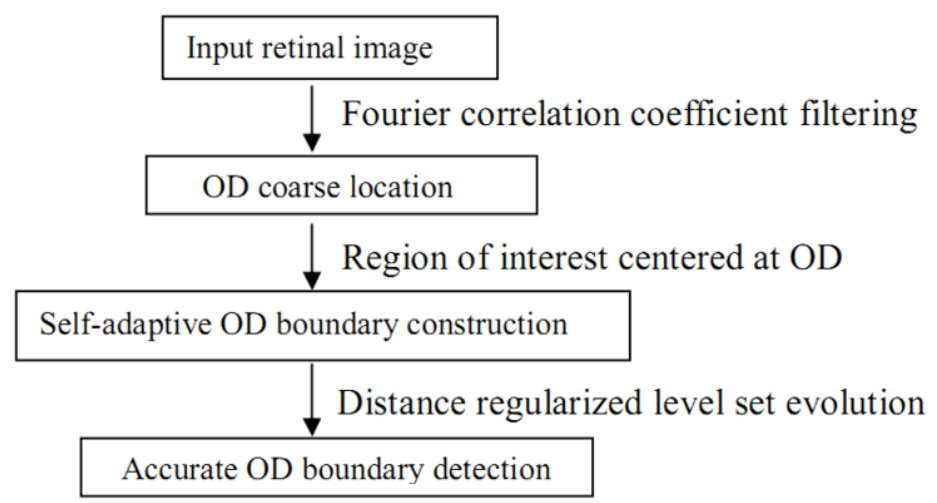

Fig. 2. The workflow chart of our proposed method.

automatically constructed for further level set evolution. In this way, an accurate OD boundary was achieved. The implementation workflow of our proposed system was illustrated as follows (Figure 2).

\subsection{OD coarse location using Fourier correlation coefficient filtering}

In this study, an isotropic Gaussian smoothing filter was used as Eq. (1):

$$
G(x, y)=\frac{1}{2 \pi \sigma^{2}} e^{-\frac{x^{2}+y^{2}}{2 \sigma^{2}}}
$$

A discrete approximation to the Gaussian function was constructed (radius was set to 18 , and scale factor was set to 0.03 ) as a template for further image pre-processing. Then, Fourier transform was performed on tested images to calculate Fourier correlation coefficients of tested images and the template. Then, the coefficients were obtained, and 3D plotting of the coefficients' matrix was shown in Figure 3A. The OD center was shown in the peak and different edges were obtained with different threshold values (Figure 3B), which could be used as coarse OD segmentation results.

After above coarse automatic localization of and OD edge detection, an initial square region of interest (ROI) centered at above localized OD center (Figure 4A) and with size larger than the diameter of coarsely segmented OD was constructed as the beginning contour for further level set algorithm (Figure 4B). The coordinate's information of such ROI in the whole tested image was preserved for further usage. 


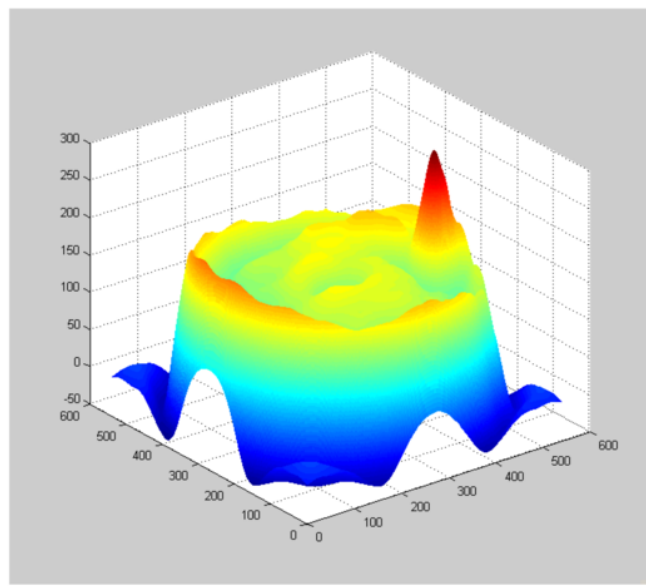

A

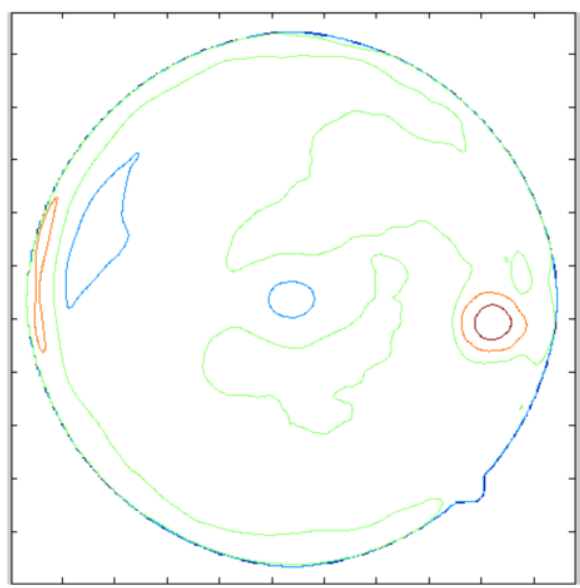

B

Fig. 3. 3d view of the Fourier correlation matrix (left) and set of contours at different levels (right).

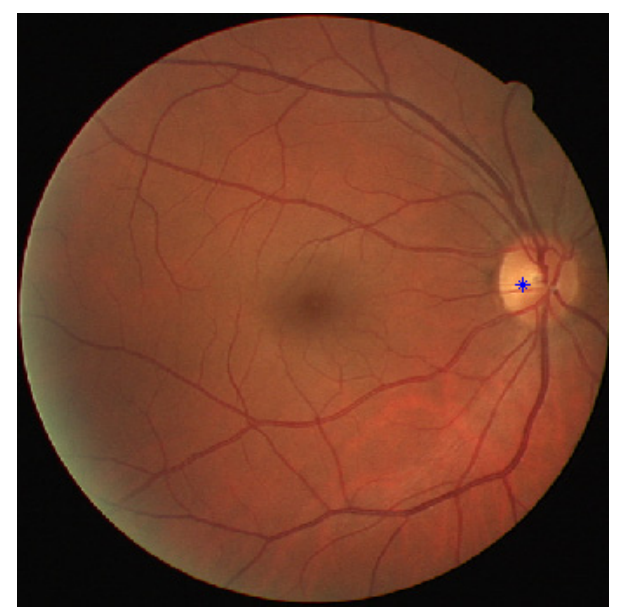

A

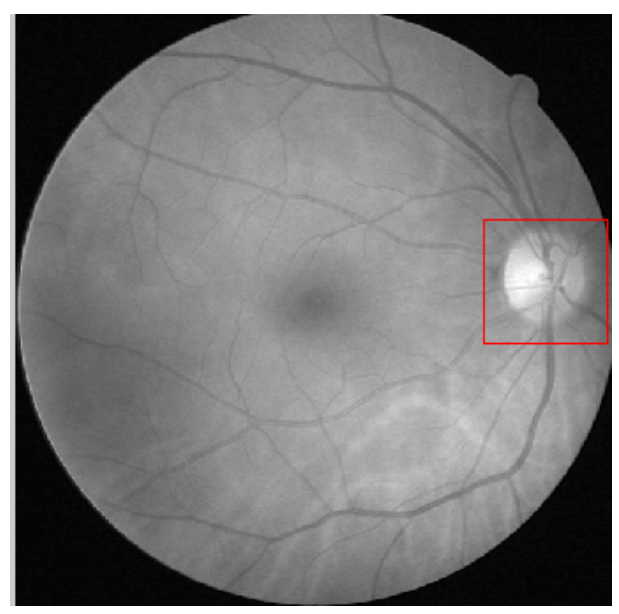

$\mathrm{B}$

Fig. 4. The pre-localization of optic center (4A) and initial contour for level set algorithm (4B).

\subsection{Distance regularized level set evolution}

Level set method was introduced by Caselles et al. [13] and Malladi et al. [14] in the context of gemetric active contour models [15] for image segmentation.

Herein, a time-dependent level set model is formulated as Eq. (2):

$$
\mathrm{L}=\frac{\partial(x, y, t)}{\partial t}=\mathrm{F}|\nabla(\mathrm{x}, \mathrm{y})|
$$

where $\mathrm{F}$ is the speed function that controls the motion of the iterated contours. 
In order to prevent irregularities of a level set function (LSF) during the level set evolution, Li et al. [16] proposed a distance regularization term, which maintains a signed distance profile near the zero level set. For this modification, the regularity of the LSF is kept by a forward-and-backward diffusion derived from the distance regularization term in the level set evolution.

In this study, an energy function $\mathrm{E}(\mathrm{L})$ was defined as Eq. (3):

$$
\mathrm{E}(\mathrm{L})=\mu \mathrm{R}(\mathrm{L})+\mathrm{E}_{\text {ext }}(\mathrm{L})
$$

in which $\mathrm{L}$ represents the level set function, $\mathrm{R}$ is the level set regulation term referred to as a distance regularization term for its role of maintaining the signed distance property of the LSF, $\mu$ is a positive constant, and $\mathrm{E}_{\mathrm{ext}}$ is the external energy. The $\mathrm{R}$ term is further defined as Eq. (4):

$$
\mathrm{R}(\mathrm{L})=\int p(\nabla L) d x
$$

in which $\mathrm{p}$ is an energy density function defined as Eq. (5):

$$
\mathrm{p}=\frac{1}{2}(s-1)^{2}
$$

which has $\mathrm{s}=1$ as unique minimum point. $\mathrm{E}_{\mathrm{ext}}$ is designed when the zero level set of level set function $\mathrm{L}$ is located at the real OD edges. In this way, we could achieve the parameters of level set evolution equation by minimization of energy function.

After iteration, the contour of OD was identified and calibrated to the original image input coordinate, and the pixels in it were preserved as 1, while the pixels out it were changed to 0 to form a binary image. Then, we quantified and compared the accuracy of the OD segmented region against the hand-labeled ground truth produced by a clinician. A region-based quantitative analysis is used to as a measure to match between two regions in the images, which access the performance of our proposed method with Eq. (6).

$$
\text { Accuracy }=\frac{T p}{T p+F p}
$$

in which $\mathrm{Tp}$, Fp are true positives, false positives respectively. Table 1 showed mean accuracy rate for all 100 images in the dataset.

Table 1

Comparison of different algorithms in OD detection

\begin{tabular}{|l|l|l|}
\hline Methods & OD detection accuracy & Speed \\
\hline Hoover [17] & $89.0 \%$ & $4 \mathrm{~min}$ \\
\hline Sinthanayothina's [18] & $96.3 \%$ & $4.5 \mathrm{~min}$ \\
\hline Foracchia [19] & $97.5 \%$ & $2 \mathrm{~min}$ \\
\hline Youssif [20] & $98.8 \%$ & $3.5 \mathrm{~min}$ \\
\hline Mahfouz [21] & $97.0 \%$ & $0.6 \mathrm{~s}$ \\
\hline Our proposed & $99.7 \%$ & $3.5 \mathrm{~s}$ \\
\hline
\end{tabular}




\section{Results}

The automatically identified OD is located correctly in 97 images out of 100 experimental images. The reason for the three mistaken images was due to the uneven background illumination, which results in the first coarse location of OD center error, changing from the actual location. In the detection of OD boundary using our contour-based model, the initial set of points that define contour of the level set were automatically selected as a square-box containing OD. Several different values were tested for the parameters of the gradient descent flow equation and with these parameter settings, the proposed algorithm was applied to 100 images in the dataset. We found that time step was set as 2; coefficient of the distance regularization term $\mathrm{R}$ was set as 0.2 ; coefficient of the weighted length term $\mathrm{L}$ was set as 2; coefficient of the weighted area term A was set as 1.5; and scale parameter in Gaussian kernel for ROI image convolution was 1.5 could achieve the best result for our images. The number of iterations for convergence is set to 200, and it always finished in 150 . The final convergent contour of OD was smooth and fit the natural edges of OD (Figures 5 and 6). The comparison of accuracy and time cost with other reported algorithms were shown in Table 1.
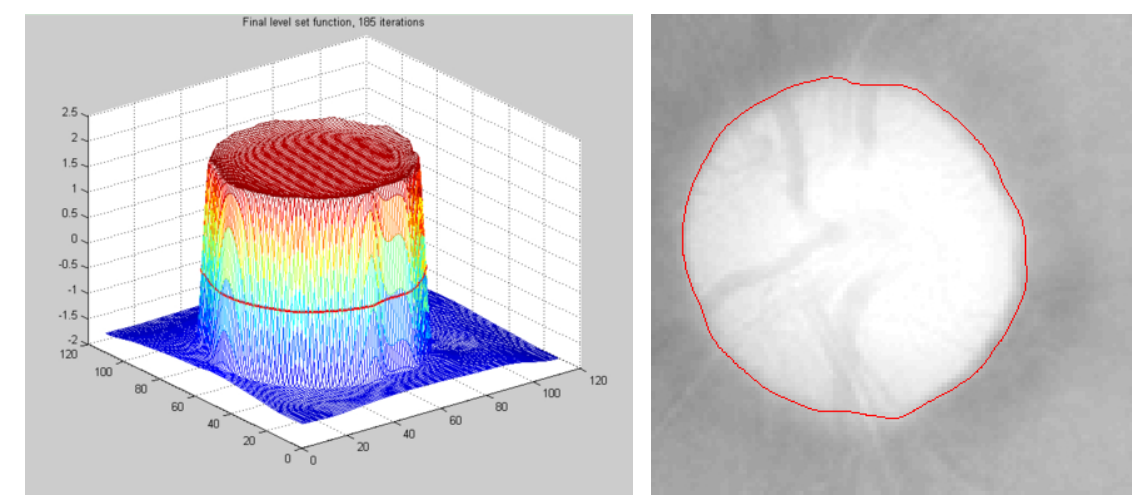

Fig. 5. Automatic segmented results (left: $3 \mathrm{~d}$ view of segmented result: right: final contour of OD).
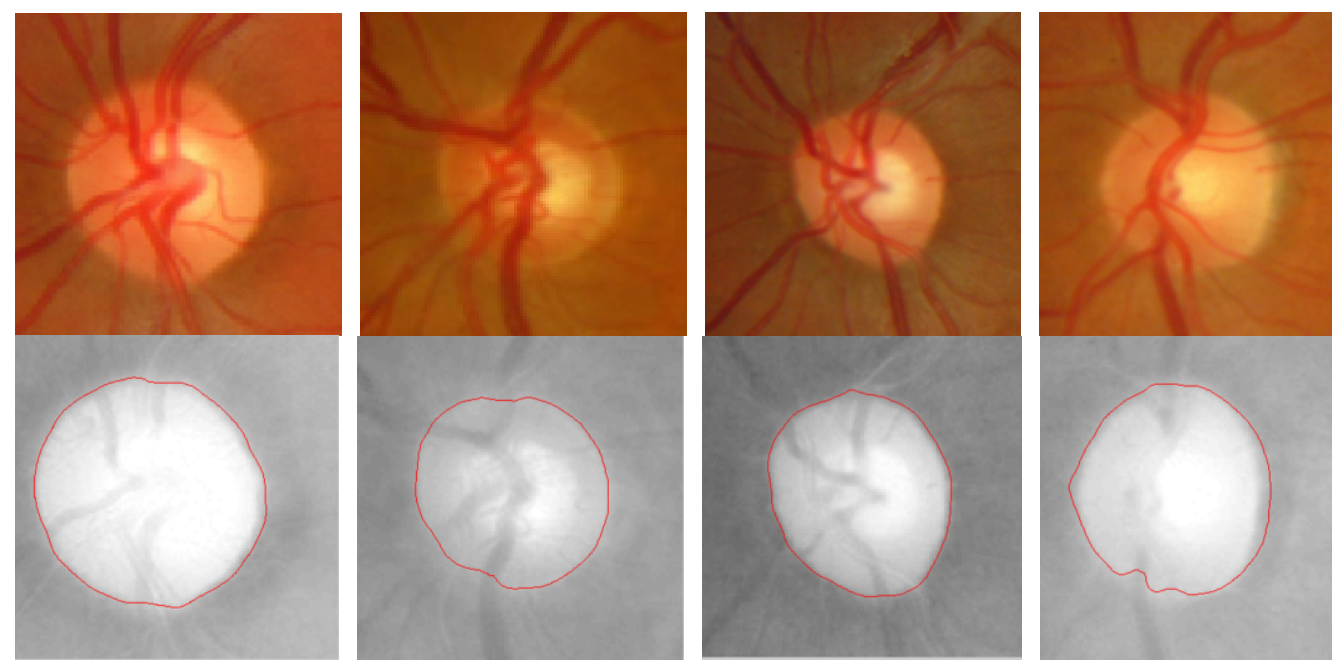

Fig. 6. The segmented results (upper: original images; lower: segmented OD contour). 


\section{Discussion and conclusion}

A number of studies have been reported on automatic OD localization and segmentation [22-25]. Normally, these approaches could be divided into two major classes: contouring modeling and pixel classification. Interestingly, a recent study compared the contour modeling method with the pixel classification method, and their results suggested that the OD region could be better determined with the contour modeling methods like active contour modeling [26].

Level set method has been selected in this study, and the initial regional curve is represented implicitly as a level set of a 2D scalar function. Different from the common edge detector or seed tracing approaches, the level set method evolves a curve by updating the level set function at fixed coordinates through time instead of tracking a curve through time. The traditional level set method has the irregularities that are developed during the level set evolution. In our study, the distance regularized level set evolution formulation could reduce iteration numbers and computation time, while maintaining sufficient numerical accuracy. A refinement of this technique is combing the coarse localization of OD to formulate an adaptive ROI as input boundary for the distance regularized level set evolution iteration. Another Gaussian smoothing kernel regularized model which penalized the level set function has been formulated by Zhang et al. [27] While Ciofolo et al. [28] proposed an automatic method for the segmentation of multiple structures with competitive level sets driven by fuzzy control.

In conclusion, our proposed self-adaptive distance regularized level set evolution method has segmented OD successfully in retinal images, without re-initialization and the need of tuning the contour parameters.

\section{Acknowledgment}

This work was supported by the grant of National Natural Science Foundation of China (No. 81271668).

\section{References}

[1] G.L. Spaeth and S.C. Reddy, Imaging of the optic disk in caring for patients with glaucoma: Ophthalmoscopy and photography remain the gold standard, Surv. Ophthalmol. 59 (2014), 454-458.

[2] H. Narasimha-Iyer, A. Can, B. Roysam, V. Stewart, H.L. Tanenbaum, A. Majerovics and H. Singh, Robust detection and classification of longitudinal changes in color retinal fundus images for monitoring diabetic retinopathy, IEEE Trans. Biomed. Eng. 53 (2006), 1084-1098.

[3] M. Niemeijer, M.D. Abramoff and B. van Ginneken, Segmentation of the optic disc, macula and vascular arch in fundus photographs, IEEE. Trans. Med. Imag. 26 (2007), 116-127.

[4] C.K. Lu, T.B. Tang, A. Laude, I.J. Deary, B. Dhillon and A.F. Murray, Quantification of parapapillary atrophy and optic disc, Invest Ophthalmol. Vis. Sci. 52 (2011), 4671-4677.

[5] E.M.F. Riverón and M. del Toro-Céspedes, Measurement of parameters of the optic disc in ophthalmoscopic color images of human retina, Lect. Notes Comput. Sci. 3287 (2004), 661-668.

[6] A. Abdel-Haleim, Y. Abdel-Razik, A.Z. Ghalwash, A.A. Sabry and G. Abdel-Rahman, Optic disc detection from normalized digital fundus images by means of a vessels' direction matched filter, IEEE. Trans. Med. Imag. 27 (2008), 11-18.

[7] M. Lalonde, M. Beaulieu and L. Gagnon, Fast and robust optic disc detection using pyramidal decomposition and Hausdorff-based template matching, IEEE Trans. Med. Imag. 20 (2001), 1193-1200.

[8] M. Foracchia, E. Grisan and A. Ruggeri, Detection of optic disc in retinal images by means of a geometrical model of vessel structure, IEEE Trans. Med. Imag. 23 (2004), 1189-1195. 
[9] J. Xu, O. Chutatape and P. Chew, Automated optic disk boundary detection by modified active contour model, IEEE Trans. Biomed. Eng. 54 (2007), 473-482.

[10] Y. Zheng, D. Stambolian, J. O'Brien and J.C. Gee, Optic disc and cup segmentation from color fundus photograph using graph cut with priors, Med. Image Comput. Comput. Assist Interv. 16 (2013), 75-82.

[11] K. Cemal and I. Cevat, Statistical techniques for detection of optic disc and macula and parameters measurement in retinal fundus images, Journal of Medical and Biological Engineering 31 (2010), 395-404.

[12] S. Osher and R. Fedkiw, Level set methods and dynamic implicit surfaces, Springer-Verlag, New York, 2002.

[13] V. Caselles, F. Catte, T. Coll and F. Dibos, A geometric model for active contours in image processing, Numer. Math. 66 (1993), 1-31.

[14] R. Malladi, J.A. Sethian and B.C. Vemuri, Shape modeling with front propagation: A level set approach, IEEE Trans. Pattern. Anal. Mach. Intell. 17 (1995), 158-175.

[15] M. Kass, A. Witkin and D. Terzopoulos, Snakes: active contour models, Int. J. Comput. Vis. 1 (1987), 321-331.

[16] C. Li, C. Xu, C. Gui and M.D. Fox, Distance regularized level set evolution and its application to image segmentation, IEEE Trans. Image Process. 19 (2010), 3243-3254.

[17] A. Hoover and M. Goldbaum, Locating the optic nerve in a netinal image using the fuzzy convergence of the blood vessels, IEEE Trans. Med. Imag. 22 (2003), 951-958.

[18] C. Sinthanayothina, J.F. Boycea, H.L. Cookb and T.H. Williamsonb, Automated localisation of the optic disc, fovea, and retinal blood vessels from digital colour fundus images, Br. J. Ophthalmol. 83 (1999), 902-910.

[19] M. Foracchia, E. Grisan and A. Ruggeri, Detection of optic disc in retinal images by means of a geometrical model of vessel structure, IEEE Trans. Med. Imag. 23 (2004), 1189-1195.

[20] A. Youssif, A.Z. Ghalwash and A. Ghoneim, Optic disc detection from normalized digital fundus images by means of a vessels' direction matched filter, IEEE Trans. Med. Imag. 27 (2008), 11-18.

[21] A.E. Mahfouz and A.S. Fahmy, Fast localization of the optic disc using projection of image features, IEEE Transactions on Image Processing 19 (2010), 3285-3289.

[22] M.D. Abramoff, W.L.M. Alward, E.C. Greenlee, L. Shuba, C.Y. Kim, J.H. Fingert and Y.H. Kwon, Automated segmentation of the optic disc from stereo color photographs using physiologically plausible features, Invest. Ophthalmol. Vis. Sci. 48 (2007), 1665-1673.

[23] M. Lalonde, M. Beaulieu and L. Gagnon, Fast and robust optic disc detection using pyramidal decomposition and Hausdorff-based template matching, IEEE Trans. Med. Imaging 11 (2001), 1193-1200.

[24] H. Li and O. Chutatape, Automated feature extraction in color retinal images by a model based approach, IEEE Trans. Biomed. Eng. 51 (2004), 246-254.

[25] J. Lowell, A. Hunter, D. Steel, A. Basu, R. Ryder and E. Fletcher, Optic nerve head segmentation, IEEE Trans. Med. Imaging 23 (2004), 256-264.

[26] C. Muramatsu, T. Nakagawa, A. Sawada, Y. Hatanaka, T. Hara, T. Yamamoto and H. Fujita, Automated segmentation of optic disc region on retinal fundus photographs: Comparison of contour modeling and pixel classification methods, Comput. Methods Programs Biomed. 101 (2011), 23-32.

[27] K. Zhang, L. Zhang, H. Song and W. Zhou, Active contours with selective local or global segmentation: A new formulation and level set method, Image Vision Computing 28 (2010), 668-676.

[28] C. Ciofolo and C. Barillot, Atlas-based segmentation of 3D cerebral structures with competitive level sets and fuzzy control, Medical Image Analysis 13 (2010), 456-470. 